\title{
Brownian motion
}

\author{
Giorgio Parisi
}

"I did not believe that it was possible to study the Brownian motion with such a precision."
From a Ietter from Albert Einstein to Jean Perrin (1909).

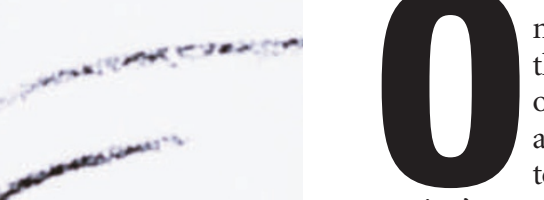

n 30 April 1905, Einstein completed his doctoral thesis on osmotic pressure, in which he developed a statistical theory of liquid behaviour based around the existence of molecules. This work, together with his subsequent paper on 'brownian motion', constitutes one of the most important, but often overlooked, contributions that Einstein made to physics.

In the closing decades of the nineteenth century, theoretical physics was in a state of turmoil. The big outstanding questions of that time have been much discussed (including within this collection). Such questions culminated in relativity and quantum mechanics - theoretical developments in which Einstein's key role is being justly celebrated this year. But it should not be forgotten that Robert Brown's seemingly innocuous observations of the irregular motions of a suspension of pollen grains in water - now known as brownian motion - also heralded a revolution in physical thought.

Although the concepts of atoms and molecules are now universally accepted, this was not the case at the turn of the twentieth century. Ludwig Boltzmann's statistical interpretation of the laws of thermodynamics - a body of work deeply rooted in the ensemble dynamical motion of material atoms - had many adherents. But there were also many heavyweight dissenters (for a time including Max Planck), who did not accept that thermodynamics had its origins in the reversible motion of invisible hypothetical particles. And many distinguished physicists of the time (among them Wilhelm Roentgen) suspected that brownian motion indicated a clear failure of Boltzmann's formulation of the second law of thermodynamics.

It was in this context that Einstein's explanation for brownian motion made an initial impression. In particular, Einstein showed that the irregular motion of the suspended particles could be understood as arising from the random thermal agitation of the molecules in the surrounding liquid: these smaller entities act both as the driving force for the brownian fluctuations (through the impact of the liquid molecules on thelarger particles), and as a means of damping these motions (through the viscosity experienced by the larger particles). This connection between displacement, $x(t)$, and the viscosity, $\eta$, can be expressed (in one dimension) as: $\left\langle x(t)^{2}\right\rangle=$ $R T t /(3 N \pi a \eta)$, where $R$ is the universal gas constant, $N$ is Avogadro's number $\left(2 R / 3 N\right.$ is Boltzmann's constant $\left.k_{\mathrm{B}}\right), T$ is the temperature and $a$ is the radius of the suspended particles. This finding went beyond simply confirming the existence of atoms and molecules, and provided a new way of determining Avogadro's number. As Einstein himself remarked, the consequence of this relation is that one can see, directly through a microscope, a fraction of the thermal energy manifest as mechanical energy. By proving that a statistical mechanics description could explain quantitatively brownian motion, all doubts concerning Boltzmann's statistical interpretation of the thermodynamic laws suddenly faded.

But this was not the end of the story. Einstein's realization that the fluctuations responsible for the agitation of the suspended particles were the same as those responsible for the friction experienced by the particles in motion - the first example of a 'fluctuation-dissipation' theorem — had farreaching consequences for other systems in equilibrium.

Generalizing this idea to other systems was easy and it became a recurrent theme in Einstein's papers: in an equilibrated system under a small perturbation there is balance between a systematic force and a chaotic fluctuating force. This is the essence of the fluctuation-dissipation theorem at equilibrium that was later derived in full generality by Kubo.

This celebrated classical fluctuation-dissipation theorem can be proved microscopically, with the details of the proof depending on the dynamics of the system. The theorem is valid for all dynamical systems in which the Boltzmann distribution is reached at equilibrium. A further extension of the basic idea occurred as recently as the 1980s when it was realized that, in certain cases, the fluctuation-dissipation theorem was a consequence of a hidden supersymmetry and time translation invariance.

When we go out of equilibrium, the formal proof fails, and new properties and behaviour appear. In some systems, the departure from equilibrium is substantial and we must resort to different tools to analyse their behaviour. A good example is turbulence, where energy is continuously injected into the system, before cascading from one length scale to another.

But the situation is different for systems that are only slightly out of equilibrium. For example, imagine a system that cannot reach equilibrium because of high free-energy barriers (that may be of energetic or of entropic nature): this situation typically applies to disordered systems, such as spin glasses and structural glasses. Such a system will approach equilibrium slowly, by jumping from one metastable state to another, and it could remain slightly out of equilibrium forever if continually perturbed with a slowly changing external field.

In such systems we can expect a separation, by many orders of magnitude, between the microscopic time scale of the system (for example, that represented by the vibrations of individual atoms) and the macroscopic time needed to cross the barrier (for example, changes in the structure of the system itself). The system can then be considered to be essentially thermalized inside a metastable state, and so fluctuationdissipation ideas can still be applied: the slowly changing overall state of the system is considered to be a small perturbation.

Although a formal analytical solution to such behaviour has yet to be found, it is encouraging that much of the off-equilibrium behaviour of disordered systems can be understood within a fluctuation-dissipation framework that in some respects constitutes a generalization of Boltzmann's equilibrium statistical mechanics. These ideas are explored in more detail for the specific case of glasses in this issue (page 222). Giorgio Parisi is in the Department of Physics, Università di Roma La Sapienza, Piazzale Aldo Moro 5, Roma 00185, Italy.

\section{FURTHER READING}

Pais, A. Subtle is the Lord... (Oxford Univ. Press, 1982).

Kuhn, T. S. Black Body Theory and the Quantum Discontinuity 1894-1911 (Oxford Univ. Press, 1978)

Mézard, M., Parisi, G. \& Virasoro, M. A. Spin Glass Theory and Beyond (World Scientific, Singapore, 1987). 